\title{
Highly Efficient and Uniform Single Stranded DNA Immobilization Using a Phase-Transfer Catalyst*
}

\author{
Miwako Nakahara ${ }^{\dagger}$ and Takashi Inoue \\ Production Engineering Research Laboratory, Hitachi, Ltd., \\ 292 Yoshida-cho, Totsuka-ku, Yokohama 244-0817, Japan
}

Akio Yamane, Toshiaki Ito, Osamu Kogi, and Noriko Ban

Life Science Division, Hitachi Software Engineering Co., Ltd., 1-1-43, Suehiro-cho, Tsurumi-ku, Yokohama 230-0045, Japan

(Received 11 November 2005; Accepted 2 December 2005; Published 27 December 2005)

\begin{abstract}
We propose a novel single stranded DNA (ssDNA) immobilization process for improving sensitivity and reproducibility of DNA microarrays. We immobilized 50 mer 5'-amino-modified oligonucleotides by forming a covalent bond between the oligonucleotides, and phenylisothiocyanate groups tethered on the surface. We used a solution containing a phase-transfer catalyst, cetyltrimethylammonium bromide (CTAB). The amount of the immobilized oligonucleotides was estimated from fluorescence intensity of $\mathrm{Cy} 5$ dyes, which were attached to the oligonucleotides. The surface density of the immobilized oligonucleotides was also measured using X-ray reflectometry and surface plasmon resonance. We found that the addition of CTAB allowed us to increase the density and uniformity of the immobilized oligonucleotides, as well as to decrease non-specific adsorption of the oligonucleotides. The highest density and uniformity were obtained when the concentration of CTAB was nearly below the critical micelle concentration (CMC). At the concentration level close to the CMC, a CTAB monolayer formed on the surface can attract the negatively charged oligonucleotides effectively because of the positive charge of CTAB. Moreover, the CTAB layer can enhance encounter rates between the hydrophilic oligonucleotides and the hydrophobic surface with the phenylisothiocyanate groups. However, at a higher concentration level above the CMC, the density of the immobilized oligonucleotides decreases because the CTAB micelles can trap the oligonucleotides in the solution. Finally, we obtained higher hybridization signals on oligonucleotides immobilized with the CTAB solution. [DOI: 10.1380/ejssnt.2005.511]
\end{abstract}

Keywords: X-ray reflection; Catalysis; Plasmon; Surface structure; Nucleic Acids

\section{INTRODUCTION}

DNA microarrays have become a widely accepted tool for gene expression profiling and genotyping $[1,2]$. Commonly used DNA microarrays rely on the specific hybridization of unknown DNA fragments to surface-bound single-stranded oligonucleotides. Many chemical reactions have been developed for attaching oligonucleotides to solid surfaces [3]. Two major platforms are in a widespread use for the arrays. The first platform involves synthesizing oligonucleotides on solid surfaces by a photolithographic or printing method based on combinatorial chemistry. The second utilizes deposition of presynthesized oligonucleotides on the surfaces. Although in-situ synthesis has several advantages over the second platform using pre-synthesized oligonucleotides, the quality of the oligonucleotides made on a surface can be hardly assessed [4]. Using pre-synthesized oligonucleotides, on the other hand, has advantage of being assessed before their immobilization on the surface; this could facilitate quality control of the arrays. However, homogeneous immobilization of a detectable quantity of the pre-synthesized oligonucleotides and reduction of nonspecifically adsorbed oligonucleotides are necessary to obtain high sensitivity and high reproducibility of the arrays.

Rickman et al. [5] have developed reaction solu-

*This paper was presented at International Symposium on Surface Science and Nanotechnology (ISSS-4), Saitama, Japan, 14-17 November, 2005.

${ }^{\dagger}$ Corresponding author: nakahara@perl.hitachi.co.jp tion for immobilizing probe cDNAs. As a result of the study, they found that adding 3-[(3-cholamidopropyl)dimethylammonio]-1-propane sulfonate to formamide or dimethyl sulfoxide (DMSO) immobilization solution allowed increasing the amount of immobilized cDNAs as well as improving uniformity of them. Diehl et al. [6] proposed an addition of betaine (N, N, N-trimethylglycine) to spotting solution used for immobilizing cDNAs. The addition of betaine improved immobilization efficiency and homogeneity of the DNAs coated on poly-L-lysine. However, those methods presented by Rickman et al. and Diehl et al. require UV irradiation for immobilizing DNAs. We rather focused on an immobilization process without using UV irradiation. Benters et al. [7] reported a method for immobilizing oligonucleotides using pre-fabricated polyamidoamine (PAMAM) starburst dendrimers as linkers between the oligonucleotides and a substrate surface. The immobilized surface possessed high immobilization efficiency and high stability.

Here, we describe a novel method for immobilizing pre-synthesized oligonucleotides on a glass surface. The simple method allows more efficient and uniform immobilization compared with a conventional method, without using UV irradiation and complicated coating processes. We apply a method for covalent attachment of amino-modified oligonucleotides to isothiocyanate groups tethered on a silicon dioxide surface by using an immobilization solution containing a phase transfer catalyst, cetyltrimethylammonium bromide (CTAB). The immobilized oligonucleotides have been quantified using a fluorescence labeling method, X-ray reflectometry, and surface plasmon resonance (SPR). Although several other spectroscopic methods, time-of-flight secondary ion mass spec- 
trometry (TOF-SIMS), X-ray photoelectron spectroscopy (XPS), and infrared spectroscopy were introduced [8, 9], above two methods, X-ray reflectometry and SPR, expected to be suitable to quantify the immobilized oligonucleotides. The surface morphology has been analyzed with the atomic force microscope (AFM). Hybridization efficiency on the newly fabricated surface is also evaluated.

\section{EXPERIMENTAL}

\section{A. Immobilization of pre-synthesized oligonucleotides}

Substrates used for this study were $6 \mathrm{~mm} \times 10 \mathrm{~mm}$ p-type Si (100) wafers; thermal silicon dioxide of 50 $\mathrm{nm}$ thickness was formed on the Si wafers. The substrates were cleaned by immersion in $10 \mathrm{wt} \% \mathrm{NaCl}, 1 \mathrm{wt} \%$ $\mathrm{HCl}$, and ethanol subsequently, rinsed in deionized water $(>18 \mathrm{M} \Omega \mathrm{cm})$, and baked at $80^{\circ} \mathrm{C}$ for $2 \mathrm{~h}$ prior to the next silanization process. Silanization with $3 \% \gamma$ aminopropyltrimethoxysilane (APTMS; Sigma-Aldrich) in methanol was performed for 5 min at room temperature $[2,10]$. After the substrates were rinsed with methanol and dried under vacuum, they were baked at $180^{\circ} \mathrm{C}$ for 2 $\mathrm{h}$; siloxane bonds were formed between the silicon dioxide surface and the silane-coupling agents by this process [11].

The homobifunctional crosslinker of 1, 4- phenylenediisothiocyanate (PDITC Kanto Chemical Co.) was dissolved in N, N-dimethylformamide (DMF) with the concentration of $0.6 \mathrm{wt} \%$, and supplemented with pyridine $(1 \%, \mathrm{v} / \mathrm{v})[2,7,10,12,13]$. The silanized substrates were immersed in the crosslinker solution for $10 \mathrm{~h}$ at room temperature, rinsed with the solvent and methanol, and dried under vacuum. In this process, one of the isothiocyanate groups of PDITC was bound to the amino group on the silanized substrates. The other isothiocyanate groups were available to form a covalent bond with an amino-modified oligonucleotide.

Pre-synthesized 5'-amino-modified oligonucleotides of 50 mer were dissolved in $500 \mathrm{mM}$ carbonate buffer adjusted to $\mathrm{pH} 8.5$ using $\mathrm{Na}_{2} \mathrm{CO}_{3}$ and $\mathrm{NaHCO}_{3}$ [2]; the concentration of the oligonucleotides was $100 \mu \mathrm{M}$. The sequence of the oligonucleotides is 5 ' $-\mathrm{NH}_{2}$ - AGT GAA TGA TGC TCA CTT GTT GCA GTA CAA TCA TCG GGT TAA AAA ACT CA-3' purchased from Sigma Genosis. CTAB was mixed in the solution with the concentration of $0.5 \mathrm{mM}$ to $50 \mathrm{mM}$. $20 \mu \mathrm{L}$ of the solution was deposited on the entire surface of the activated substrate. The substrates were incubated in a chamber under saturated humidity at $25^{\circ} \mathrm{C}$ for $4 \mathrm{~h}$, then rinsed with $0.1 \%$ Triton $\mathrm{X}$ 100 solution, $0.05 \% \mathrm{HCl}, 1 \mathrm{M} \mathrm{KCl}$ subsequently to remove non-specifically bound oligonucleotides, and dried under vacuum. For blocking non-reacted isothiocyanate groups, the substrates were immersed in $1 \mathrm{M}$ 2-aminoethanol at $25^{\circ} \mathrm{C}$ for $1 \mathrm{~h}$ and rinsed with deionized water. Control experiments, where non-modified oligonucleotides lacking the end modification of an amino group were immobilized on the surface, were also performed to verify that the covalent bonds between the amino-modified oligonucleotides and the isothiocyanate group were formed.

\section{B. Characterization of immobilized pre-synthesized oligonucleotides}

\section{Fluorescence labeling and scanning analysis}

The 3' end of the immobilized oligonucleotides was labeled with a fluorescent 2', 3'-dideoxynucleotide triphosphates (ddNTP). Cyanine-labeled ddNTPs (ddNTP-Cy5; GE Healthcare) and terminal deoxynucleotidyl transferase (TdT; Roche) were used for the labeling [14]. The following solutions were mixed and the final concentrations are $1.25 \times \mathrm{TdT}$ reaction buffer $(50 \mathrm{mM}$ potassium cacodylate/ $6 \mathrm{mM}$ Tris-HCl/0.06 mg/ml BSA), 13\% DMSO, $3.1 \mathrm{mM} \mathrm{CoCl} 2,1 \mu \mathrm{M}$ ddATP-Cy5, 5 Unit $/ \mu \mathrm{L}$ TdT adjusted using deionized water. We spotted the solution on the entire surface of the substrate where the probe oligonucleotides were immobilized, and then incubated the substrates at $37^{\circ} \mathrm{C}$ in order to proceed with the addition reaction of ddATP-Cy 5 to the 3 ' end of the probe oligonucleotides. After that we washed the substrates using $1 \times$ SSC (Standard Saline Citrate)/0.1\%SDS (sodium dodecylsulfate) solution at $37^{\circ} \mathrm{C}$ twice, rinsed using deionized water at room temperature, and dried under vacuum.

The substrates with the fluorescence labeled probe oligonucleotides were analyzed using a confocal laser scanner (CRBio II, Hitachi Software Engineering). Fluorescence intensities were quantified by measuring emission of $\mathrm{Cy} 5$ from the surfaces of the substrates with excitation light at $635 \mathrm{~nm}$, using the fluorescence image analysis software package (DNASIS; Hitachi Software Engineering). We obtained fluorescence intensity averaged over 96 spots on each surface; the diameter of the spot is $200 \mu \mathrm{m}$. The fluorescence intensities were measured with the same laser power and photomultiplier sensitivity.

\section{X-ray reflectivity (XRR) analysis}

$\mathrm{X}$-ray reflectivity (XRR) measurements are performed to evaluate mass surface density of the immobilized oligonucleotide layer on the substrates [15] using a X-ray reflectometer (RIGAKU ATX-G) with the X-ray wavelength of $\lambda=0.154 \mathrm{~nm}\left(\mathrm{CuK}_{\alpha 1}\right)$. Specular reflectivity from the planer substrates was recorded during the measurement. Surface density of the layer was calculated using the obtained specular reflectivity curves. We fitted the obtained curves with theoretical reflectivity curves derived from Parrattfs formula. In this fitting process, we applied a suitable and simple two-layer model for the film; the one-layer was consisted of the linker layer and the oligonucleotide layer, and the other layer was the silicon dioxide layer on the silicon substrates. Since X-ray was irradiated on the entire surface of the substrate, the calculated surface density was the averaged density of the film.

\section{Surface plasmon resonance (SPR) measurement}

The amount of the immobilized probe oligonucleotides on the substrates was measured using the surface plasmon resonance (SPR) spectroscopy. For the SPR mea- 
(a)

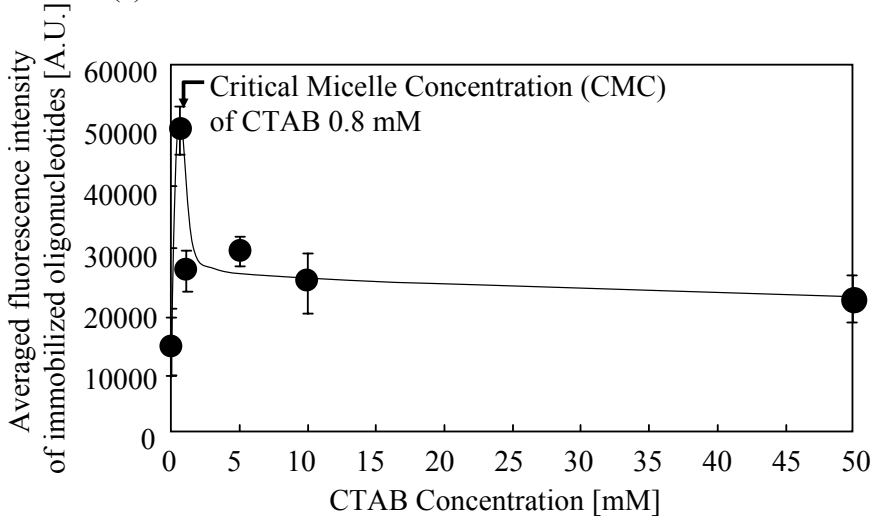

(b)

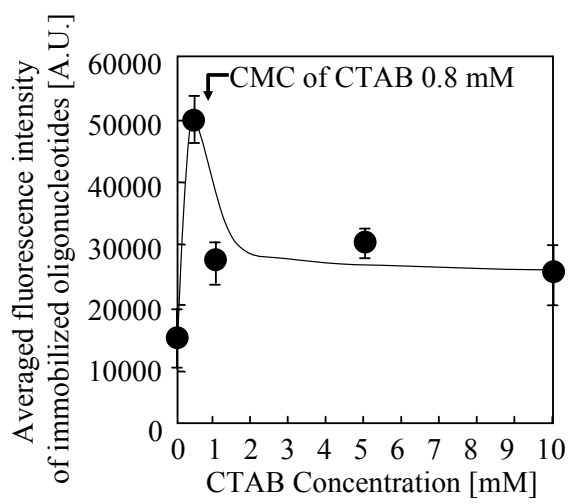

FIG. 1: Dependence of the amount of immobilized Cy5-labeled probe oligonucleotides on CTAB concentration added in the probe immobilization solution. (a) CTAB concentration from $0 \mathrm{mM}$ to $50 \mathrm{mM}$, and (b) CTAB concentration from $0 \mathrm{mM}$ to $10 \mathrm{mM}$.

surement, the sensor surfaces are created by applying a thin layer of gold $(\sim 50 \mathrm{~nm})$ to a glass surface. In the SPR unit, a light beam strikes the surface from the backside of the sensor at an angle where the beam is totally reflected. Under the condition, the evanescent wave can interact with mobile electrons at the gold film. At a particular incident angle, SPR is induced and the intensity of the reflected light is reduced [16]. The resonance angle at which this intensity of the reflected light becomes minimal is a function of the refractive index of the solution close to the gold layer. The change in angle, caused by binding or dissociation of molecules from the sensor surface, is proportional to the mass of bound material [17, 18]. We used the Biacore X (Biacore) to measure the amount of the probes. The amount was calculated from a difference of the resonance angles between the state before immobilization and that after immobilization. Usually, the surface used for the SPR detection is composed of a thin gold layer. In our case, however, the immobilization reaction is carried out on the $\mathrm{SiO}_{2}$ surface. In order to measure the immobilization reaction on the $\mathrm{SiO}_{2}$ surface, we deposited $\mathrm{SiO}_{2}$ by a sputtering process on the thin gold layer. The thickness of the $\mathrm{SiO}_{2}$ layer is $10 \mathrm{~nm}$ so that the evanescent wave can interact with the immobilized oligonucleotides, and thereby the amount of probe oligonucleotides can be detected.

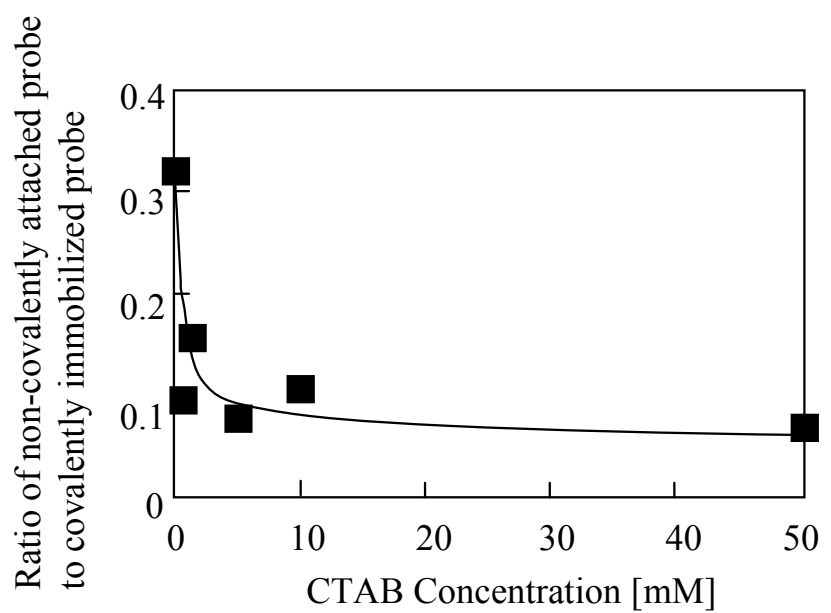

FIG. 2: Dependence of the ratio of non-covalently attached oligonucleotides (non-amino-modified oligonucleotides) to covalently immobilized oligonucleotides (amino-modified oligonucleotides) on CTAB concentration added in the probe immobilization solution. The ratios were obtained from fluorescence intensities of $\mathrm{Cy} 5$ attached to the oligonucleotides.

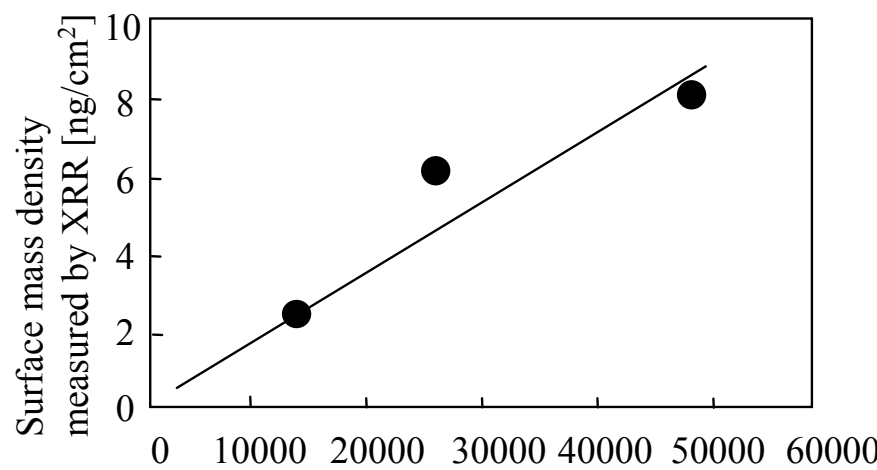

Fluorescence intensity of immobilized probe oligonucleotides [A.U.]

FIG. 3: Correlation between surface mass densities and fluorescence intensities of the probe oligonucleotides immobilized with CTAB concentration of $0 \mathrm{mM}, 0.5 \mathrm{mM}$, and $1 \mathrm{mM}$.

\section{Atomic force microscope (AFM) analysis}

The AFM images of the surfaces of the substrates were taken in air with a Dimension 3000 (Digital Instruments) operated in tapping mode. The silicon probes with a cantilever integrated with a sharp tip were used for imaging the morphology of the surfaces. Image processing analysis was performed using Nanoscope III software package. We scanned $500 \mathrm{~nm}$ square area for each measurement.

\section{Hybridization assays}

Hybridization experiments were performed using perfectly matched 50 mer target oligonucleotides with a sequence of 5'-TGA GTT TTT TAA CCC GAT GAT TGT ACT GCA ACA AGT GAG CAT CAT TCA 
TABLE I: Dependence of surface mass and molecular densities of immobilized probe oligonucleotides on added CTAB concentration. Those surface densities were measured by XRR and SPR.

\begin{tabular}{|c|c|c|c|c|}
\hline \multirow{2}{*}{$\begin{array}{c}\text { CTAB } \\
\text { concentration } \\
(\mathrm{mM}) \\
\end{array}$} & \multicolumn{2}{|c|}{$\begin{array}{c}\text { Surface density } \\
\text { by XRR measurement }\end{array}$} & \multicolumn{2}{|c|}{$\begin{array}{c}\text { Surface density } \\
\text { by SPR measurement }\end{array}$} \\
\hline & $\left(\mathrm{ng} / \mathrm{cm}^{2}\right)$ & $\left(\right.$ molecules $\left./ \mathrm{cm}^{2}\right)$ & $\left(\mathrm{ng} / \mathrm{cm}^{2}\right)$ & $\left(\right.$ molecules $\left./ \mathrm{cm}^{2}\right)$ \\
\hline 0 & 26 & $1.0 \times 10^{12}$ & 15 & $5.9 \times 10^{11}$ \\
\hline 0.5 & 81 & $3.1 \times 10^{12}$ & 39 & $1.5 \times 10^{12}$ \\
\hline 1.0 & 63 & $2.4 \times 10^{12}$ & - & - \\
\hline
\end{tabular}

CT-3'. The target oligonucleotides were dissolved in $5 \times \mathrm{SSC} / 0.5 \% \mathrm{SDS}$ solution. The surface of the substrates was hybridized with the $1 \mu \mathrm{M}$ solution of the target oligonucleotides at $42^{\circ} \mathrm{C}$ for $48 \mathrm{hr}$. After the hybridization, fluorescence intensities from the substrates were measured using the laser scanner, and were quantified from emission of Cy5. We obtained fluorescence intensity averaged over 96 spots on each surface; the diameter of each spot was $200 \mu \mathrm{m}$. The fluorescence intensities were measured with the same layer power and photomultiplier sensitivity.

\section{RESULTS AND DISCUSSION}

\section{A. Quantification of immobilized probes}

Results demonstrating the effect of the surfactant CTAB concentration on the amount of covalently immobilized probe oligonucleotides were obtained using the fluorescence Cy5 labeling and fluorescence scanning analysis (Fig. 1). The results show that the addition of CTAB in the probe immobilization solution allows increasing the amount of immobilized probe oligonucleotides. We found a maximal point where the CTAB concentration was around $0.5 \mathrm{mM}$, close to the critical micelle concentration (CMC) [19] of CTAB (Fig. 1(b)). Above the CMC, the amount of the immobilized oligonucleotides decreased with an increase in the CTAB concentration (Figs. 1(a), (b)). In order to confirm that the obtained fluorescence intensities represent covalently bound oligonucleotides, we also quantified non-covalently attached probe oligonucleotides using the non-modified oligonucleotides as a control experiment. Figure 2 shows the ratio of the noncovalently attached oligonucleotides to the covalently immobilized oligonucleotides. The ratio was decreased with an increase in CTAB concentration, reaching below 0.1 . From the results, it is obvious that an addition of CTAB increased covalently bound probe oligonucleotides on the substrate.

The surface densities of the immobilized probe oligonucleotides were measured using X-ray reflectometry. Table I shows surface mass densities of the immobilized oligonucleotides obtained from the X-ray reflectivity (XRR) data. We estimated the surface mass densities of the oligonucleotides by subtracting surface mass density of only the linker layer measured with XRR, $1.4 \times 10^{2} \mathrm{ng} / \mathrm{cm}^{2}$, out of the mass densities of the layer including both the linker and oligonucleotide layer. The fluorescence intensities of the immobilized probe oligonucleotides were correlated with the surface mass densities (Fig. 3). The addition of CTAB with the concentration nearly below the CMC allows us to increase the immobilized probe oligonucleotides by more than three times. Although XRR measurement is suitable for quantifying an ultra-thin layer on flat surfaces, heterogeneous and uneven layers such as polymer layers could be difficult to analyze quantitatively. Therefore, we also measured the immobilized probe oligonucleotides using SPR measurement. Table 1 also shows the surface densities of the probe oligonucleotides immobilized with the CTAB concentration of $0 \mathrm{mM}$ and $0.5 \mathrm{mM}$, obtained by SPR. The surface densities are calculated with the change in the surface plasmon resonance angle, which represents mass of the immobilized molecules; a shift of 0.001 degree is roughly equivalent to a change in the mass of $1 \mathrm{pg} / \mathrm{mm}^{2}$ for biomolecules [16]. The result obtained from the XRR measurement was consistent with the result from the SPR, although the values from XRR were slightly larger than those from SPR. In this XRR measurement, the sample surface contained the blocking molecules of 2-aminoethanol together with the immobilized oligonucleotides. The blocking molecules could increase the surface density. From these results, we estimated probe oligonucleotide arrangement on the surface where the probe oligonucleotides were immobilized with the CTAB concentration of $0.5 \mathrm{mM}$. If we assume that the oligonucleotide is uniformly distributed in a grid array format, the distance between one probe oligonucleotide and the nearest neighbor of the probe oligonucleotide could be around from $6 \mathrm{~nm}$ to $8 \mathrm{~nm}$.

Figure 4 shows morphology of the surface with the immobilized probe oligonucleotides, measured with AFM. We found that the surface where the probe oligonucleotides were immobilized at the CTAB concentration of $0.5 \mathrm{mM}$ had relatively corrugated configuration compared with other surfaces. The corrugated spots are expected to represent immobilized oligonucleotides.

We obtained uniformity of the immobilized probe oligonucleotides by calculating coefficients of variation (Cv) among 96 spots where the oligonucleotides with the same 50 mer sequence were immobilized at the same CTAB concentration. Dependence of the uniformity on CTAB concentration is shown in Fig. 5. We found that the highest uniformity was obtained when the CTAB concentration level was close to the CMC. Consequently, adding CTAB of an appropriate concentration was effective for increasing the uniformity of the probe oligonucleotides as well as increasing the probe densities. 
(a)

(c)

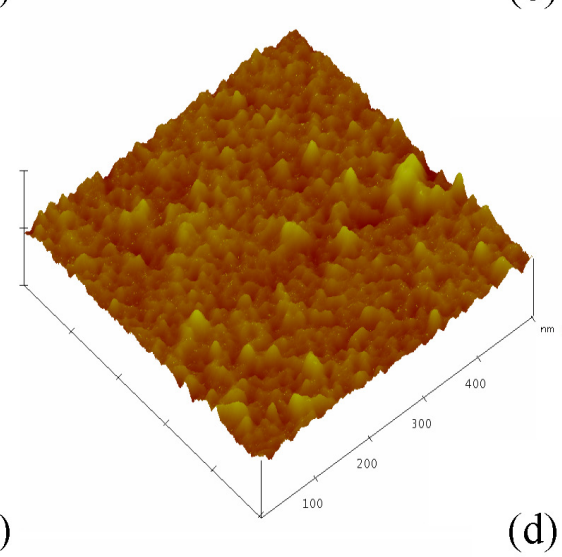

(b)

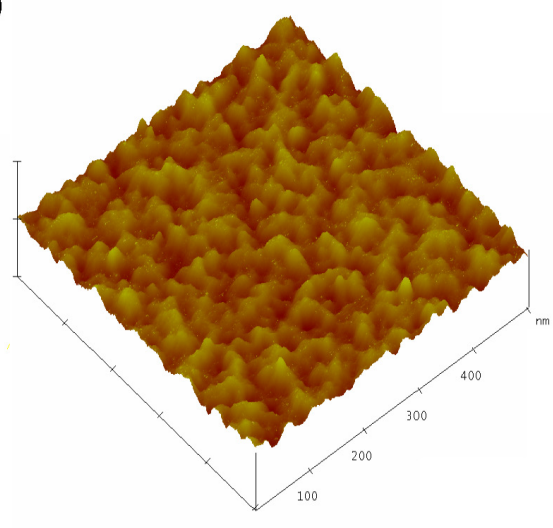

(d)
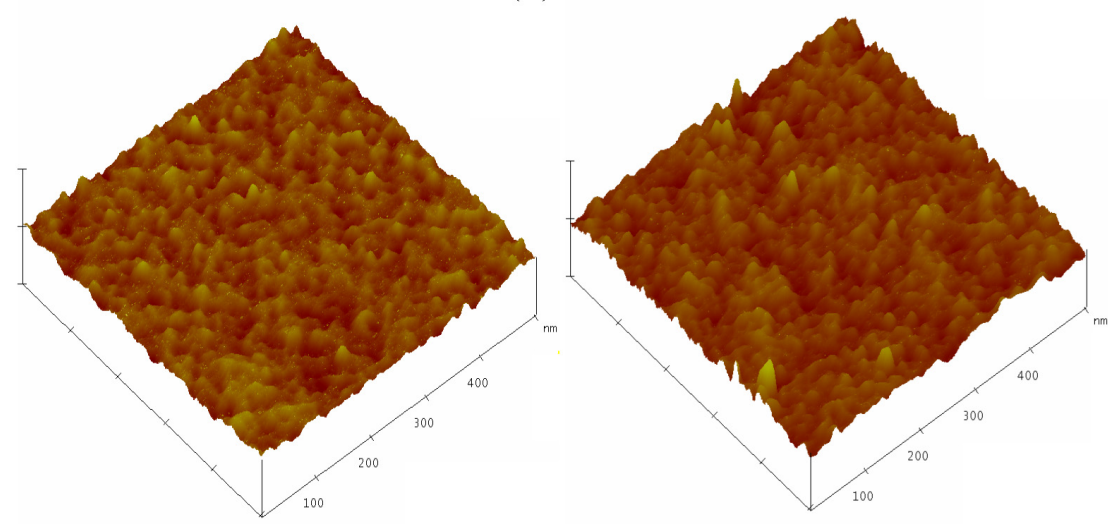

FIG. 4: Morphology of surfaces with and without immobilized probe oligonucleotides. The probes were immobilized (a) without CTAB, (b) with CTAB concentration of $0.5 \mathrm{mM}$, and (c) with CTAB concentration of $5.0 \mathrm{mM}$. (d) Silanized surface without immobilized probe oligonucleotides. The figure image size is $500 \mathrm{~nm}$ square area, and z range is $10 \mathrm{~nm}$.

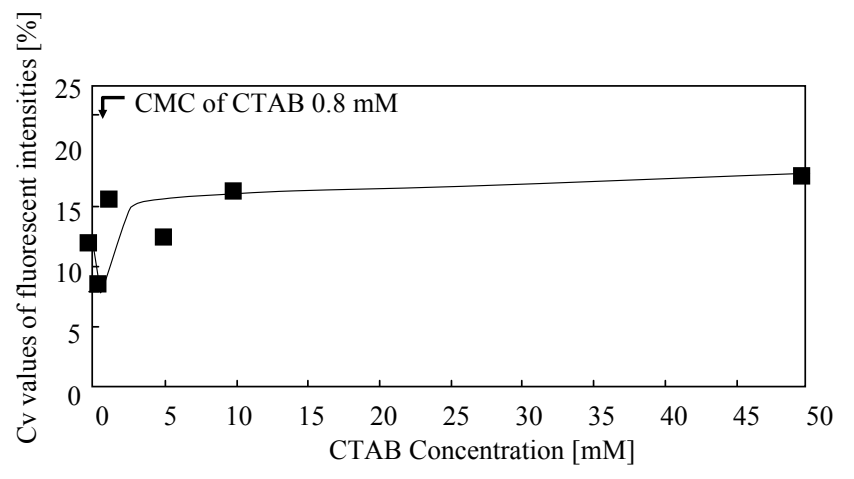

FIG. 5: Dependence of $\mathrm{Cv}$ values of 96 spots where probe oligonucleotides were immobilized on CTAB concentration added in the probe immobilization solution.

\section{B. Immobilization reaction mechanism}

We discuss mechanism of the oligonucleotide immobilization reaction in the condition of adding the surfactant CTAB. Surfactant can work as a phase transfer catalyst, since the surfactant accelerates transferring ions between water phase and oil phase. The catalyst increases frequency of the collision between molecules dissolved in the water phase and oil phase. Therefore, the reaction with the ions between two phases can be enhanced. In our case, the probe oligonucleotides represent anionic ions and immobilization solution represents the water phase. The substrate surface with the hydrophobic phenylisothiocyanate groups represents the oil phase. The surfactant of CTAB can increase collision frequency between the probe oligonucleotides in the water (hydrophilic) phase and the isothiocyanate groups in the oil (hydrophobic) phase, resulting in enhancement of reaction efficiency of the probe immobilization. In addition, whereas the oligonucleotides are negatively charged, CTAB is positively charged. If CTAB is adsorbed on the substrate, the flux of the oligonucleotides to the surface can be increased due to the Coulombic interaction.

Figure 6(a) shows the schematic structure of CTAB, and Fig. 6(b) shows a schematic representation for explaining mechanism how the amount of the immobilized probe oligonucleotides is increased, in the case where the CTAB is added to the concentration at the vicinity of the CMC. CMC is a concentration where the surface tension of the solution against the surfactant concentration posses discontinuity. When the surfactant concentration is equal or less than the CMC, the surfactant molecules exist in a form of a monomer in the solution or on the substrate surface. When approaching the CMC, the surfactant adsorbs as a single layer on a substrate surface. Thus, the CTAB may adsorb as a single layer on the substrate surface having phenylisothiocyanate groups. The CTAB layer facili- 
(a)

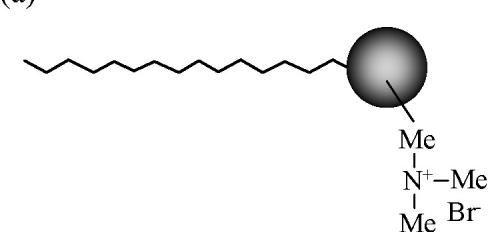

(c)

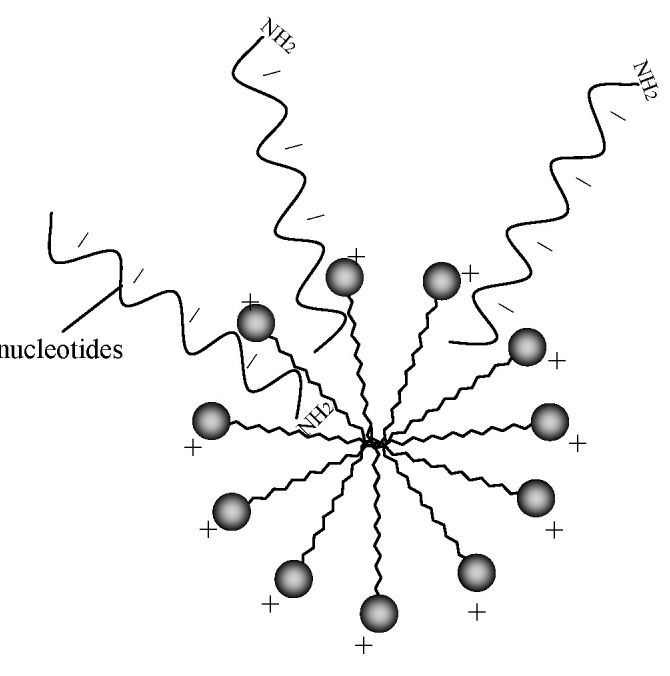

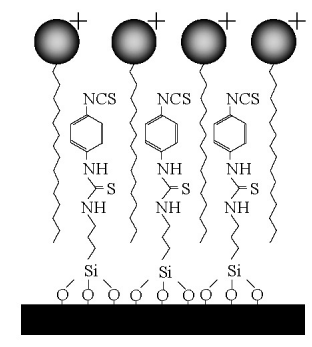

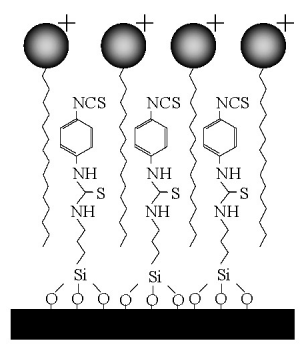

FIG. 6: A schematic representation for explaining immobilization reaction mechanism with CTAB. (a) represents CTAB molecular structure. The circular part is hydrophilic, while the tail part is hydrophobic. (b) Surface during the immobilization reaction when the CTAB concentration is close to the CMC. (c) Surface during the immobilization reaction when the CTAB concentration is above the CMC.

tates negatively charged probe oligonucleotides to be adsorbed on the surface as shown in Fig. 6(b). In addition, the CTAB acts as a phase transfer catalyst, which accelerates inter-molecular encounter rates between the hydrophobic surface and the hydrophilic oligonucleotides.

On the other hand, around the region of the CMC, behavior of the surfactant molecules changes drastically. The CTAB molecules aggregate into micelle forms. When the concentration is more than the CMC, CTAB micelles were created as shown in Fig. 6(c). The micelle may trap the probe oligonucleotides in the solution, thereby decreasing concentration of the probe oligonucleotides near the surface. Therefore, the micelle creation of CTAB may work toward blocking the reaction between the probe oligonucleotides and the reactive isothiocyanate groups on the surface. However, although the reaction efficiency decreased above the CMC concentration, the reaction efficiency was higher than the condition where CTAB is not added. Furthermore, there is a feature that the amount of the immobilized probe oligonucleotides is stable in the concentration region equal to or more than the $\mathrm{CMC}$, even if the concentration of the surfactant is changed. This feature could have advantage for building a robust process.

Since the addition of CTAB improved uniformity of the immobilized probe oligonucleotides, it is assumed that CTAB can help the oligonucleotides to diffuse on the surface uniformly during the immobilization process. Moreover, it is also assumed that CTAB can enable the non-

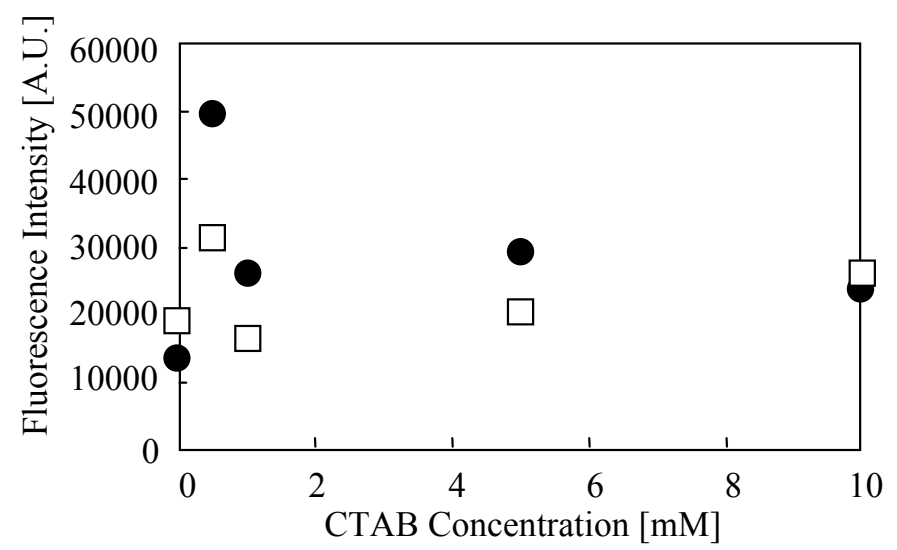

FIG. 7: Dependence of fluorescence intensity of Cy5-labeled hybridized targets on CTAB concentration added in the probe immobilization solution. Open squares represent fluorescence intensities of hybridized targets, while closed circles represent fluorescence intensities of the immobilized Cy5-labeled probe oligonucleotides.

specifically adsorbed oligonucleotides to be removed during the washing process after the immobilization, from the result that the addition of CTAB reduced non-specifically adsorbed probe oligonucleotides. 


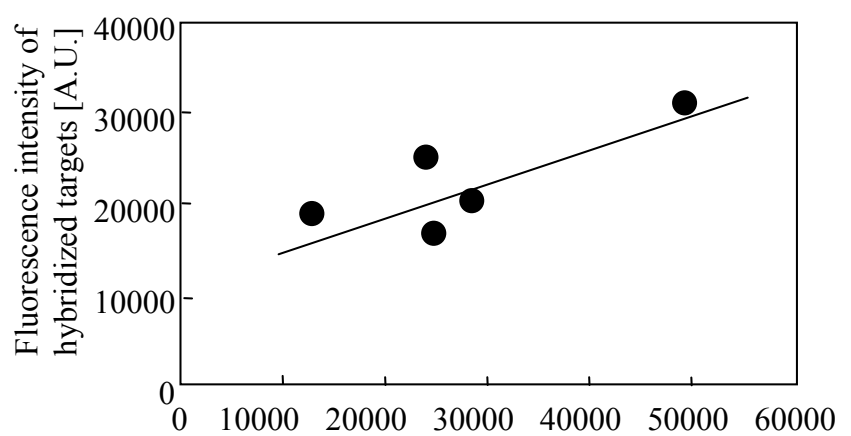

Fluorescence intensity of the immobilized probe oligonucleotides [A.U.]

FIG. 8: Correlation between fluorescence intensity of Cy5labeled hybridized targets and that of the immobilized $\mathrm{Cy} 5$ labeled probe oligonucleotides.

\section{Hybridization assays}

Quantity of hybridization to the immobilized probe oligonucleotides was evaluated. The target oligonucleotides whose sequences are perfectly matched with the probe oligonucleotide were hybridized on the substrate. The terminal of the complete complementary target oligonucleotide is modified with a fluorescent molecule of Cy5. Figure 7 shows fluorescence intensities of the hybridized targets detected by the fluorescence scanner. We found that higher hybridization fluorescence signals were obtained if the probe oligonucleotides were immobilized in the solution with the CTAB concentration close to the CMC. Figure 8 shows a correlation between fluorescence intensities from the probe oligonucleotides with Cy5 modified using TdT, and fluorescence intensities from the hybridized targets with Cy5. The fluorescence intensities from the probe oligonucleotides were approximately proportional to those from the hybridized targets. It is obvious that probe oligonucleotide immobilization with an addition of CTAB contributes increase in hybridization efficiency.

\section{CONCLUSIONS}

An addition of CTAB to probe ssDNA immobilization solution allows us to increase efficiency and uniformity of the immobilized ssDNA as well as to reduce nonspecifically adsorbed ssDNAs. The amount of immobilized probe oligonucleotides was examined with the fluorescence detection and $\mathrm{XRR}$, and $\mathrm{SPR}$. With the results, the immobilization reaction mechanism was discussed. The highest immobilization efficiency and uniformity were obtained when the CTAB concentration level was nearly close to the CMC. At the concentration level slightly lower than the CMC, a CTAB monolayer formed on the surface can attract the negatively charged oligonucleotides effectively because of the positive charge of CTAB. Moreover, the CTAB that is a phase transfer catalyst can enhance molecular interaction between the hydrophilic oligonucleotides and the hydrophobic surface with the phenylisothiocyanate groups. However, at a higher concentration level above the $\mathrm{CMC}$, the surface density of the immobilized oligonucleotides decreases since the CTAB micelles can trap the oligonucleotides in the solution. Using the surface where the probe oligonucleotides were immobilized in a solution containing CTAB, we obtained higher hybridization signals.

\section{Acknowledgments}

We are grateful to Dr. Yasuo Komatu (National Institute of Advanced Industrial Science \& Technology), Mr. Takashi Sugimoto (Hitachi Software Engineering), Dr. Yoshinobu Kohara and Dr. Hideyuki Noda (Central Research Laboratory at Hitachi), Dr. Tomonori Saeki (Production Engineering Research Laboratory at Hitachi) and Ms. Akika Niiyama for fruitful discussions.
[1] T. R. Hughes, et al., Nature Biotechnology 19, 342 (2001).

[2] Z. Guo, R. A. Guilfoyle, A. J. Thiel, R. Wang, and L. M. Smith, Nucleic Acid Research 22, 5456 (1994).

[3] M. C. Pirrung, Angew. Chem. Int. Ed. 41, 1276 (2002).

[4] E. Southern, K. Mir, and M. Shchepinov, Nature Genetic Supplement 21, 5 (1999).

[5] D. S. Rickman, C. J. Herbert, and L. P. Aggerbeck, Nucleic Acid Research 31, e109 (2003).

[6] F. Diehl, S. Grahlmann, M. Beier, and J. D. Hoheisel, Nucleic Acid Research, 29, e38 (2001).

[7] R. Benters, C. M. Niemeyer, D. Drutschmann, D. Blohm, and D. Wohrle, Nucleic Acid Research, 30, 10 (2002).

[8] L. Cheran, D. Vukovich, and M. Thompson, Analyst 128, 126 (2003).

[9] G. Shen, M. F. G. Anand, and R. Levicky, Nucleic Acid Research, 32, 5973 (2004).

[10] P. T. Charles, G. J. Vora, J. D. Andreadis, A. J. Fortney, C. E. Meador, C. S. Dulcey, and D. A. Stenger, Langumuir 19, 1586 (2003).
[11] E. T. Vandenberg, L. Bertilsson, B. Liedberg, and I. Lundström, Journal of Colloid and Interface Science, 147, 103 (1991).

[12] R. Möller, A. Csáki, J. M. Köhler, and W. Fritzsche, Nucleic Acid Research, 28, 91 (2000).

[13] R. Benters, C. M. Niemeyer, and D. Wöhrle, Chembiochem 2, 686 (2001).

[14] K. Lindroos, U. Liljedahl, M. Raitio, and A. Syvänen, Nucleic Acid Research, 29, 69 (2001).

[15] G. Pirri, F. Damin, M. Chiari, E. Bontempi, and L. E. Depero, Anal. Chem. 76, 1352 (2004).

[16] W. D. Wilson, Science 295, 2103 (2002).

[17] E. Stenberg, B. Person, H. Roos, and C. Urbaniczky, Journal of Colloid and Interface Science 143, 513 (1990).

[18] B. Johnsson, S. Löfas and G. Lindquist, Analytical Biochemistry 198, 268 (1991).

[19] K. P. Ananthapadmanabhan, E. D. Goddard, N. J. Turro, and P. L. Kuo, Langmuir 1, 352 (1985). 